\title{
Peripheral blood stem cell transplants
}

\author{
J L Byrne, N H Russell
}

\section{Autologous peripheral blood stem cell transplantation}

High dose myeloablative treatment followed by autologous haemopoietic stem cell rescue is increasingly used in the treatment of haematological malignancies and solid tumours with the aim of achieving a dose-response effect. Apart from the risk of disease relapse, the major drawback of this approach is the risk of procedure related toxicity reflecting the period of pancytopenia. The past decade has witnessed a number of important advances in the field of autologous transplantation with a resultant reduction in this toxicity. The most important of these has been the gradual switch to peripheral blood as a source of haemopoietic stem cells. This has not only led to a profound acceleration in haemopoietic reconstitution, but has also extended the indications of autologous transplantation to situations in which autologous bone marrow transplantation would not have been considered because of toxicity. In view of these advantages, it is no surprise that the use of peripheral blood as a source of haemopoietic progenitors has almost replaced bone marrow in the autologous setting, increasing from $15 \%$ of all European autologous transplants in 1991 to $79 \%$ in $1995 .{ }^{1}$

\section{Mobilisation of peripheral blood stem cells}

The origins of peripheral blood stem cell (PBSC) transplantation date from the early 1960 s when it was realised that peripheral blood contained stem cells that could give rise to all three haemopoietic cell lineages in irradiated mice. In the resting state, relatively few stem cells circulate in the peripheral blood. This number can be increased up to 100 -fold in the recovery phase following myelosuppressive chemotherapy, ${ }^{2}$ and can be further increased by the addition of haemopoietic growth factors. ${ }^{3}$ These cells can readily be collected by continuous flow cell separation and enumerated either by colony unit forming assay (CFU-GM) or by flow cytometric analysis of the cells expressing the CD34 antigen.

The ability of PBSC transplants to produce rapid engraftment is dependent on the number of CD34+ cells infused. We and others have shown that patients receiving more than $2.5 \times$ $10^{6} \mathrm{CD} 34+$ cells/kg experienced rapid neutrophil and platelet engraftment, while those receiving fewer cells showed evidence of delayed haemopoietic recovery, particularly affecting platelets. ${ }^{4}$

The optimal mobilisation regimen to maximise the number of circulating progenitor cells has yet to be determined. For autologous transplants a combination of chemotherapy and a growth factor such as G-CSF (filgrastim) is usually employed, since these combinations have been shown to result in a superior CD34+ stem cell yield compared with G-CSF alone. ${ }^{5}$ Gianni et al described the use of cyclophosphamide at a dose of $7 \mathrm{~g} / \mathrm{m}^{2}$ together with GM-CSF. ${ }^{5}$ However, high doses of cyclophosphamide have been associated with a significant morbidity and even mortality, and various groups ${ }^{46}$ have now reported that the use of lower doses, ranging from $1.5 \mathrm{~g} / \mathrm{m}^{2}$ to $4 \mathrm{~g} / \mathrm{m}^{2}$ combined with G-CSF can mobilise sufficient PBSC for successful engraftment in the majority of patients. These lower doses of cyclophosphamide are probably not as efficient at mobilising CD34+ stem cells as the higher doses previously used, but this is countered by the reduced toxicity of the mobilisation schedule. Combination chemotherapy regimens such as epirubicin, ifosphamide, and etoposide (IVE) have recently been shown to be superior to intermediate dose cyclophosphamide for stem cell mobilisation. ${ }^{7}$ Failure to mobilise sufficient PBSC may occur and is usually related to previous heavy exposure to chemotherapy, especially alkylating agents, ${ }^{8}$ or to radiotherapy, particularly to the pelvis. Combinations of cytokines, such as G-CSF plus stem cell factor or G-CSF plus megakaryocyte growth and development factor, hold considerable promise for the future.

Advantages of PBSC transplantation over autologous bone marrow transplantation The main advantage of mobilised PBSC over bone marrow for autologous transplantation is the consistent acceleration of haemopoietic reconstitution observed. In 1992, Sheridan et al showed that patients receiving G-CSFmobilised PBSC together with autologous bone marrow showed a highly significant acceleration of platelet engraftment compared with historical controls (15 v 39 days). ${ }^{9}$ A similar enhancement of haematological recovery was reported by Jones et al in a study of 26 patients, 18 of whom received PBSC alone. ${ }^{10}$ These patients also had a significant reduction in the number of blood products transfused, the number of febrile days, days on antibiotics, and days in hospital compared with historical bone marrow transplant controls. This benefit of PBSC transplantation over bone marrow transplantation in terms of faster haemopoietic reconstitution has now been confirmed in a randomised study of lymphoma patients. ${ }^{11}$ The speed of neutrophil recovery may be further enhanced by the use of post-transplant G-CSF, ${ }^{12}$ although no further reduction in the duration of thrombocytopenia or transfusion requirements has been observed, and it is not yet clear whether their use leads to a survival benefit. 
In addition to the ease of PBSC collection which can be performed as an outpatient procedure without the need for general anaesthetic, another advantage of PBSC transplantation is the ability to collect PBSC in patients where previous irradiation to the pelvis precludes marrow harvest, thus extending the use of high dose therapy to a wider range of patients. $^{2}$ In addition there is a theoretical advantage of using PBSC for transplantation in cases where there is significant marrow tumour cell infiltration, although recent studies have in fact shown a high rate of circulating tumour cells in both lymphoma ${ }^{13}$ and myeloma patients. ${ }^{1415}$ Nevertheless in patients with minimal marrow disease, there is some evidence that PBSC harvests may contain less tumour contamination than marrow, since studies of clonal Ig gene rearrangements using molecular techniques have often given positive results on marrow while PBSC harvests remain negative. ${ }^{16}$ Whether the use of PBSC will translate into an increased survival compared to the use of bone marrow for autologous transplantation remains unclear. Autologous bone marrow transplantation does carry a significant mortality risk in heavily pretreated patients, as exemplified by the $13 \%$ transplant related mortality seen in the MRC-AML-10 trial. ${ }^{17}$ Certainly the rapid haemopoietic engraftment is advantageous in extending the age range for high dose therapy and in our experience patients over 60 years of age can tolerate PBSC transplantation as well as younger adults.

Recent advances have led to the commercial availability of reliable techniques to purify CD34+ cells by positive selection. These purified cell populations have been shown to effectively reconstitute haemopoiesis following high dose therapy. The major aim of CD34 selection is to "positively purge" the stem cell transplant, since the CD34 antigen is only expressed on immature haemopoietic cells and not on tumour cells. Current trials are under way to compare such CD34+ selected with unselected transplants in both myeloma and lymphoma patients.

Finally, economic considerations suggest that despite the increased cost of collection of PBSC compared with marrow, the more rapid engraftment kinetics leads to a reduction in the duration of hospital admission and antibiotic and blood product usage, resulting in a cost-benefit advantage for PBSC transplantation over autologous bone marrow transplantation.

\section{Clinical indications of PBSC transplantation}

The major application of high dose therapy with PBSC support in the United Kingdom has been in the treatment of lymphoproliferative disorders including myeloma and lymphoma. The median survival of patients with multiple myeloma remains at about 30 months with few long term survivors living more than 10 years. Newer chemotherapy combinations have not had a major impact on these outcomes despite producing higher response rates.

High dose melphalan without stem cell support was pioneered in the early 1980s and showed high response rates with about $30 \%$ of patients achieving complete remission. However, these responses were not sustained and considerable toxicity resulted from the myelosuppression. Subsequently dose escalation of melphalan was achieved using autologous marrow rescue to reduce the duration of cytopenia and this led to improved response rates with reduced morbidity. A large randomised study has shown a significant survival advantage for high dose melphalan with bone marrow support compared with conventional chemotherapy. ${ }^{18}$ Substitution of blood stem cells for autologous marrow in patients undergoing autologous transplants for myeloma is an attractive option owing to the proven benefits in terms of rapid engraftment and the potential reduction in tumour cell contamination, and is undergoing direct comparison with autologous bone marrow transplantation in a study organised by the French Myeloma Intergroup. PBSC can be mobilised successfully in myeloma patients provided that previous high exposure to alkylating agents has not occurred. ${ }^{19} 20$

Since cells carrying the same IgH rearrangement as the myeloma clone are frequently detected in peripheral blood harvests using polymerase chain reaction (PCR) techniques, ${ }^{15}$ this raises the possibility that relapse following PBSC transplantation is caused by reinfusion of potentially malignant cells. As a consequence, strategies using positive CD34+ selection before blood stem cell transplantation as a means of purging are being evaluated in prospective randomised studies, including one organised by the European Group for Blood and Marrow transplantation (EBMT).

In lymphoma, evidence for a clinical benefit for high dose compared with conventional treatment in patients with lymphoma has been difficult to obtain, as few large randomised studies have been performed. One of the few studies that has shown superiority in terms of progression-free survival for the transplant regimen compared to lower dose conventional salvage treatment is the BNLI study of BEAM versus mini-BEAM, which confirmed that dose intensity is important in the treatment of relapsed Hodgkin's disease. ${ }^{21}$ Approximately $50-60 \%$ of patients given high dose therapy at chemosensitive first relapse experience prolonged disease-free survival. ${ }^{22}$ Thus intensive treatment requiring haemopoietic stem cell support has become an accepted therapeutic option for patients with Hodgkin's disease relapsing after previous combination chemotherapy.

Sufficient numbers of PBSC can be obtained in the majority of patients, and provided that adequate numbers of CD34+ cells are infused, autologous transplantation using PBSC is an effective low risk strategy for relapsed or refractory Hodgkin's disease.

Similarly PBSC transplantation has largely replaced autologous bone marrow transplantation as a safe and effective treatment for 
patients with non-Hodgkin's lymphoma. ${ }^{1}$ Here, there is more controversy over which patients are likely to benefit; however, one large randomised study has shown a survival benefit of transplantation over conventional treatment. ${ }^{23}$ In general this treatment is reserved for patients with chemosensitive relapsed high or intermediate grade nonHodgkin's lymphoma, but is being evaluated as intensified treatment for patients with high risk features. Although the rapid engraftment observed using PBSC has led to reduced toxicity of this procedure, an overall benefit of PBSC transplantation over autologous bone marrow transplantation has not been proven. An EBMT analysis of matched patients with non-Hodgkin's lymphoma undergoing PBSC transplantation versus autologous bone marrow transplantation showed no survival difference, while another study has shown better survival rates for a subgroup of patients with aggressive non-Hodgkin's lymphoma when PBSC were reinfused instead of unpurged marrow. $^{24}$ In Hodgkin's disease a recent matched pair analysis carried out by the EBMT showed a slight survival benefit for patients receiving bone marrow; however, follow up remains short. ${ }^{25}$

High dose treatment with stem cell rescue is now increasingly being used in the treatment of low grade lymphoma, although this approach is made more problematic by the high frequency of tumour cell contamination. Follicular lymphoma, for example, is a heterogeneous disorder with a variable clinical course; however, patients with advanced stage disease treated with standard chemotherapy have an overall survival of $30-40 \%$ at five to 10 years. $^{26}$ Cure is rarely achieved by conventional chemotherapy, whereas long term remissions have been reported even in patients in the poor prognosis group following high dose therapy and autologous bone marrow transplantation, ${ }^{27}$ particularly where the marrow has been successfully purged of contaminating lymphoma cells. PBSC transplants are increasingly being used to support high dose therapy in patients with follicular lymphoma. However, assessment using PCR for the $\mathrm{t}(14 ; 18)$ molecular marker shows that PBSC products are frequently contaminated with lymphoma cells, even when the bone marrow is in apparent morphological remission. ${ }^{28}$ PBSC harvests contain significantly fewer CD19+ and CD34/ CD19 coexpressing cells than are present in the bone marrow, suggesting that chemotherapy-mobilised PBSC may provide the optimal starting material for manipulations such as CD34+ selection to reduce tumour contamination. ${ }^{28}$ Using the technique of CD34+ selection we have found that the PBSC harvests can be successfully purged in about two thirds of cases, although additional purging strategies such as CD19+ depletion may be required for complete removal of contaminating lymphoma cells. ${ }^{28}$

Clearly what is needed in order to answer the question of whether PBSC transplantation offers a survival advantage over bone marrow in lymphoproliferative diseases are large ran- domised trials; however, these will be increasingly difficult to mount as most transplant groups have switched to PBSC to support high dose therapy. ${ }^{1}$

\section{Allogeneic PBSC transplantation}

The first allogeneic PBSC transplants were conducted in the early 1990s and were facilitated by the ability of haemopoietic growth factors to mobilise stem cells into the peripheral blood. From data reported to international registries such as the IBMTR it has been estimated that more than 2500 such transplants have been performed worldwide. The majority of these transplants were from HLA identical siblings, but PBSC transplantation are increasingly being performed from mismatched family donors and unrelated donors. The major issues relating to the use of PBSC for allogeneic transplantation have involved donor mobilisation, engraftment kinetics, and the incidence of graft versus host disease.

Donor mobilisation has almost entirely been with G-CSF, which has generally been recognised as having a low toxicity profile although serious adverse events have been reported following donor mobilisation, ${ }^{29}$ for example one recently reported case of splenic rupture occurring in a family donor undergoing mobilisation with G-CSF. ${ }^{30}$ Two other adverse events related to PBSC donation by normal donors have also been described. ${ }^{29}$ Serious adverse events have, however, also been reported after bone marrow harvesting of normal donors, ${ }^{31}$ and whether one procedure is intrinsically safer than the other is unclear. However, although G-CSF is well tolerated by the majority of normal donors there may be some situations where it should be considered with caution. These include donors with a history of autoimmune disease, inflammatory disorders, venous thrombosis, atherosclerosis, or a previous malignancy treated by chemoradiotherapy. ${ }^{29} \mathrm{~A}$ four day course of G-CSF at a dose of $10 \mu \mathrm{g} / \mathrm{kg} / \mathrm{d}$ is an effective regimen and has been recommended by investigators within the EBMT. ${ }^{32}$ Using this dose of G-CSF, the white cell count typically reaches $50-60 \times 10^{9}$ \% litre and CD34+ cells peak in the peripheral blood by day $4-5$. In a recent analysis of 44 donors undergoing PBSC mobilisation in the United Kingdom, a median of $5.75 \times 10^{6} / \mathrm{kg}$ CD34+ cells was collected with a median of two leucaphereses, and only in two donors were less than $2 \times 10^{6} / \mathrm{kg} \mathrm{CD} 34+$ cells collected. ${ }^{33} \mathrm{In}$ 35 donors undergoing leucapheresis we have found the use of peripheral venous access adequate and have not had to resort to femoral or central venous cannulation; however, we take care to assess donor venous access before harvesting and would recommend bone marrow harvesting if venous access is poor. Finally the possible long term effects of G-CSF are unclear and therefore long term follow up of donors who have been given this agent would be advisable.

The experience of several reported series is that haemopoietic engraftment following allogeneic PBSC transplantation is accelerated and 
this has now been confirmed in a randomised trial organised by the EBMT. ${ }^{34}$ As is the case for autologous PBSC transplantation, the kinetics of engraftment appear to be related to the number of CD34+ cells infused in that we found that patients receiving less than $4 \times$ $10^{6} / \mathrm{kg}$ had slower engraftment, comparable with that seen following allogeneic transplantation using bone marrow. ${ }^{33}$

One of the major initial concerns over using PBSC for allogeneic transplantation related to the possibly increased risk of graft versus host disease (GVHD), owing to the significantly increased number of $\mathrm{T}$ lymphocytes infused in PBSC harvests-these are approximately 10fold greater than in bone marrow. However, clinical experience of allogeneic PBSC transplants has failed to show any significant differences in the incidence or severity of acute GVHD, ${ }^{32}$ although some reports have suggested an increased risk of chronic GVHD. The explanation for the lack of severe acute GVHD may reflect differences in the cellular composition of PBSC harvests, which contain large numbers of monocytes and CD4-/ CD8- natural suppressor cells, both of which have been reported to suppress in vitro GVHD reactions. The latter cell populations appear to be mobilised from the bone marrow during G-CSF treatment ${ }^{35}$ and secrete large amounts of Th2-type cytokines, including interleukin-4.

Other potential advantages of the use of G-CSF-mobilised PBSC which are currently being clinically investigated relate to the potential for a superior graft versus leukaemia effect, which might reduce relapse rate after transplantation. There is some evidence from a murine transplantation model that the relapse risk is reduced by using PBSC, and a recent case-control study of patients at the MD Anderson Hospital receiving PBSC has suggested a significantly lower relapse rate, ${ }^{36}$ a result confirmed in an IBMTR analysis. Whether this is really the case will have to await the results of the randomised trials of PBSC compared with bone marrow for allogeneic transplantation, which are now underway.

The clinical use of allogeneic PBSC has now extended beyond matched sibling transplants to mismatched family member and unrelated donor transplantation. Evidence has been produced to suggest that the high doses of CD34+ cells that can be collected by G-CSF mobilisation can overcome graft rejection which has been a major problem with mismatched transplants. The experience of PBSC in unrelated donor transplantation remains limited at this time, particularly in the United Kingdom where a moratorium on the administration of G-CSF to volunteer donors has been imposed (although this is currently under review). However, the limited data that are available suggest that the incidence and severity of GVHD is similar to that with bone marrow transplantation. The potential for the use of PBSC in unrelated donor transplantation is exciting, particularly as cell dose has been shown to be a major determinant of outcome. ${ }^{37}$ Thus data from Seattle have shown that patients who received a transplant containing more than $3.85 \times 10^{8} / \mathrm{kg}$ mononuclear cells had a significantly improved outcome. This improvement was because of a reduction in nonleukaemic deaths rather than a reduced risk of relapse, and may reflect a more rapid haematological and immune reconstitution associated with high cell dose. As it has been shown that both haematological and possibly immune reconstitution are more rapid following allogeneic PBSC transplantation in HLA matched sibling donors, the use of PBSC may have a major impact in improving the outcome following unrelated donor transplantation.

\section{Future directions}

The use of PBSC in both autologous and allogeneic transplantation is rapidly increasing . New strategies for their use are being evaluated for solid tumours and autoimmune diseases as well as for haematological malignancies. Allogeneic PBSC programmes from volunteer unrelated donors are being started. Improvements in mobilisation schedules, stem cell isolation, and purification procedures and techniques for both positive and negative purging to reduce tumour cell contamination or to deplete $\mathrm{T}$ lymphocytes are emerging. The possibility of ex vivo stem cell expansion to further accelerate haemopoietic recovery or to provide sufficient stem cells in a single mobilisation to support several cycles of high dose therapy is under investigation. In addition, techniques by which new genetic material is introduced into the stem cell population are being developed and may lead to the cure of various inherited diseases by somatic gene therapy. Thus it appears that the rapid evolution in the use of PBSC transplantation witnessed over the past decade is set to continue well into the future.

1 Gratwohl A, Hermans J, Baldomero H. Blood and marrow transplantation activity in Europe 1995. Bone Marrow transplantation activity in

2 Kessinger HH, Armitage JO, Smither DM, et al. High dose therapy and autologous peripheral blood stem cell transplantation for patients with lymphoma. Blood 1989; transplantation

3 Duhrsen U, Villeval JL, Boyd J, et al. Effects of recombinant granulocyte colony stimulating factor on hematopoietic progenitor cells in cancer patients. Blood 1988;72:2074-81.

4 Haynes A, Hunter A, McQuaker G, et al. Engraftment characteristics of peripheral-blood stem-cells mobilized with cyclophosphamide and the delayed addition of G-CSF. Bone Marrow Transplant 1995;16:359-63.

5 Gianni AM, Siena S, Bregni M, et al. Granulocytemacrophage colony-stimulating factor to harvest circulating haemopoietic stem cells for autotransplantation. Lancet 1989;ii:580-5.

6 Jones HM, Jones SA, Watts MJ, et al. Development of a simplified single-apheresis approach for peripheral-blood progenitor-cell transplantation in previously treated paprogenitor-cell transplantation in previously treated pa-

7 McQuaker G, Haynes AP, Stainer C, et al. Stem cell mobilisation in resistant or relapsed lymphoma - superior yield of progenitor cells following a salvage regimen comprising ifosphamide, etoposide and epirubicin compared to intermediate dose cyclophosphamide. Br f Haematol 1997; 98:228-33.

8 Haas R, Mohle R, Fruhauf S, et al. Patient characteristics associated with successful mobilizing and autografting of peripheral blood progenitor cells in malignant lymphoma. Blood 1994;83:3787-94.

9 Sheridan WP, Begley CG, Juttner CA, et al. Effect of peripheral-blood progenitor cells mobilised by filgrastim therapy. Lancet 1992;339:640-4.

10 Jones $\mathrm{H}$, Jones S, Watts $\mathrm{M}$, et al. Development of a simplified single apheresis approach for peripheral blood progenitor cell transplantation in previously treated patients with cell transplantation in previously treated
lymphoma. F Clin Oncol 1993;12:1693-6. 
11 Schmitz N, Linch DC, Dreger P, et al. A randomised trial of filgrastim-mobilised peripheral blood progenitor cell transplantation versus autologous bone marrow transp

$12 \mathrm{McQuaker}$ I, Hunter A, Pacey S, et al. Low dose filgrastim significantly enhances neutrophil recovery following autologous PBSCT in patients with lymphoproliferative disorders: evidence for clinical and economic benefit. $\mathcal{F}$ Clin Oncol 1997;15:451-7.

13 Lambrechts A, Hupkes P, Dorssers L, et al. Translocation $(14 ; 18)$-positive cells are present in the circulation of the majority of patients with localised (stage I and II) follicular non-Hodgkin's lymphoma. Blood 1993;82:2510-15.

14 Bird JM, Bloxham D, Samson D, et al. Molecular detection of clonally rearranged cells in peripheral blood progenitor cell harvests from multiple myeloma patients. $\mathrm{Br} \mathcal{F} \mathrm{Haema}-$ tol 1994;88:110-16.

15 Owen R, Haynes AP, Evans PA, et al. PCR assessment of peripheral blood progenitor cell harvest (PBPCH) conperipheral blood progenitor cell harvest (PBPCH) contamination and Cliferection in multiple myeloma and Pathol 1996;149:112-17.

16 Langlands K, Craig J, Parker A. Molecular determination of minimal residual disease in peripheral blood stem cell harvests. Bone Marrow Transplant 1990;5:64-5.

17 Burnett A, Goldstone A, Stevens R, et al. Role of BMT in addition to intensive chemotherapy in AML in 1st CR Results of the MRC AML10 trial. Blood 1994;84:2529-31.

18 Attal M, Harrousseau J, Stoppa A, et al. A prospective randomised trial of autologous bone marrow transplantation and chemotherapy in multiple myeloma. $N$ Engl f Med 1996;335:91-7.

19 Demirer T, Buckner C, Gooley T, et al. Factors influencing collection of peripheral blood stem cells in patients with multiple myeloma. Bone Marrow Transplant 1996;17:937-41.

20 Tricot G, Jagannath S, Vesole D, et al. Peripheral blood stem cell transplants for multiple myeloma: identification of favourable variables for rapid engraftment in 225 patients. Blood 1995;85:588-96.

21 Linch C, Winfield D, Goldstone A, et al. Dose intensification with autologous bone marrow transplantation in tion with autologous bone marrow transplantation in relapsed and resistant Hodgkin's disease: re

22 Chopra R, McMillan A, Linch D, et al. The place of high dose BEAM therapy and autologous bone marrow transplantation in poor risk Hodgkin's disease. A single centre eight year study of 155 patients. Blood 1993;81: $1137-45$

23 Philip T, Gugielmi C, Hagenbeck A, et al. Autologous bone marrow transplantation as compared with salvage chemotherapy in relapses of chemo-sensitive NHL. $N$ Engl $7 \mathrm{Med}$ 1995;333:1540-5.

24 Vose J, Anderson J, Kessinger A, et al. High dose chemotherapy and autologous haematopoietic stem cell transplantation for aggressive non-Hodgkin's lymphoma. $\mathcal{f}$ Clin Oncol 1993;11:1846-51.
25 Majolino I, Pearce R, Taghipour G, et al. Peripheral blood stem cell transplantation versus autologous bone marrow lymphomas: a new matched pair analysis of the European group for blood and marrow transplantation. $\mathcal{F}$ Clin Oncol 1997;15:509-17.

26 Horning S. Treatment approaches to the low grade lymphomas. Blood 1994;83:881-4.

27 Freedman A, Ritz J, Neuberg D, et al. Autologous bone marrow transplantation in 66 patients with a history of low grade B-cell non-Hodgkin's lymphoma. Blood 1991;77: 2524-9.

28 McQuaker I, Haynes A, Anderson S, et al. Engraftment and molecular monitoring of CD34+ PBSC transplants for follicular lymphoma: a pilot study. f Clin Oncol 1997;15: 2288-95.

29 Anderlini P, Korbling M, Dale D, et al. Allogeneic blood stem cell transplantation: considerations for donors. Blood 1997;90:903-8.

30 Becher P, Wagler M, Matous S, et al. Spontaneous splenic rupture following administration of granulocyte-colonystimulating factor (G-CSF) occurring in an allogeneic donor of peripheral blood stem cells. Biol Blood Marrow Transplant 1997;3:45-9.

31 Buckner CD, Peterson FB, Bolonesi BA. Bone marrow donors. In: Forman SJ, Blume KG, Thomas ED, eds. Bone marrow transplantation. Boston: Blackwell Scientific Publications, 1994.

32 Russell NH, Gratwohl A, Schmitz N. The place of blood stem cells in allogeneic transplantation. $\mathrm{Br} \mathcal{F}$ Haematol 1996;93:747-53.

33 Miflin G, Russell NH, Hutchinson RM, et al. Allogeneic peripheral blood stem cell transplantation for haematological malignancies - an analysis of engraftment and GVHD risk. Bone Marrow Transplant 1997;19:9-13.

34 Schmitz N, Bacigalupo A, Hasenclever D, et al. Allogeneic bone marrow transplantation versus filgrastim-mobilised peripheral blood progenitor cell transplantation in patients with early leukaemia - results of a randomised multicentre trial. Bone Marrow Transplant (in press).

35 Zeng D, DejbakshJones S, Strober S, et al. Granulocyte colony-stimulating factor reduces the capacity of blood mononuclear cells to induce graft versus host disease: impact on blood progenitor cell transplantation. Blood 1997;90:453-63.

36 Korbling M, Mirza N, Thall P, et al. HLA-identical allogeneic blood stem cell transplantation: the MD Anderson Cancer Centre experience [abstr]. Bone Marrow Transplant 1997;19(suppl 1):284a.

37 Sierra J, Storer B, Hansen J, et al. Transplantation of marrow cells from unrelated donors for treatment of high risk acute leukaemia: the effect of leukaemic burden, donor HLAmatching and marrow cell dose. Blood 1997;89:4226-35. 